\title{
Multi-Envelope Precoding for Massive MIMO Systems
}

\author{
Mücahit Gümüş, Student Member, IEEE, and Tolga M. Duman ${ }^{(0)}$, Fellow, IEEE
}

\begin{abstract}
Constant envelope (CE) precoding is a recently proposed technique for massive MIMO systems that decreases the hardware complexity of base station circuitry. In this letter, we build on this idea and propose a multi-envelope precoding method, which utilizes more than one (but only a few, e.g., 2 or 3 ) envelope levels and assigns higher levels on the antenna group(s) requiring more power in order to increase the achievable rates. The results demonstrate substantial gains with respect to the $\mathrm{CE}$ approach for both cases of discrete and continuous phase shifters with almost no increase in hardware complexity and a small increase in computational complexity.
\end{abstract}

Index Terms-Multi-envelope precoding, constant envelope precoding, massive MIMO, large scale MIMO.

\section{INTRODUCTION}

$\mathbf{M}$ ASSIVE MIMO techniques employing hundreds of base station (BS) antennas in order to serve simultaneously tens of users through the same time and frequency resources have recently become a promising and active research area due to their potential in $5 \mathrm{G}$ and beyond wireless systems [1]. It has been shown that massive MIMO achieves much higher data rates and offers improved power efficiencies [2]. However, the use of a large number of antennas results in significantly increased hardware complexity, in particular, for the standard implementation for which the number of (highly linear and expensive) power amplifiers (PAs) needed is the same as the number of BS antennas. Motivated by the need for reducing the hardware costs, constant envelope (CE) precoding techniques have been proposed [3]-[4].

In CE precoding, the transmitted signal is generated by varying only the phase of the constant amplitude baseband symbols, hence the peak-to-average-power-ratio (PAPR) is highly improved, which makes utilizing highly nonlinear, power efficient and very cheap PAs applicable in BS circuitry. Analog or digital phase shifters (PSs) can be utilized, and it can be shown that massive MIMO gains are still possible for flat [3], [4] or even frequency selective fading channels [5].

While the hardware costs are reduced, the CE precoding suffers from a performance loss with respect to the average power constraint (APC) precoding. For instance, the $\mathrm{CE}$ precoding needs approximately $1.7 \mathrm{~dB}$ more $\mathrm{BS}$ power for an average achievable information rate of 2 bits per channel use (bpcu) when $200 \mathrm{BS}$ antennas serve 40 mobile terminals over a Rayleigh fading channel [3]. In order to

Manuscript received January 12, 2018; accepted March 4, 2018. Date of publication March 14, 2018; date of current version October 11, 2018. The associate editor coordinating the review of this paper and approving it for publication was S. K. Mohammed. (Corresponding author: Tolga M. Duman.)

The authors are with the Department of Electrical and Electronics Engineering, Bilkent University, 06800 Ankara, Turkey (e-mail: mgumus@ee.bilkent.edu.tr; duman@ee.bilkent.edu.tr).

Digital Object Identifier 10.1109/LWC.2018.2815679 remedy this problem, in this letter, we propose a novel multi-envelope precoding (MEP) technique for massive MIMO systems utilizing more than one but only a few (e.g., 2-3) envelope levels, which decreases the required additional power and increases the achievable data rates with respect to $\mathrm{CE}$ precoding. Our basic approach is as follows: we first group the antennas by utilizing the zero-forcing (ZF) criterion to determine which antenna is to be assigned to which envelope level. Then, we compute the required phase for each antenna's signal by modifying the methods in [3] and [4] appropriately. In a way, we aim at approaching the APC precoding solution by using only a few envelope levels.

In the proposed MEP approach, PAs work on 2-3 saturation points (to be able to implement the 2-3 different signal envelopes needed as opposed to a single envelope in $\mathrm{CE}$ precoding). We also note that, based on our results, these predefined envelope levels can be picked close to each other with a close to optimal performance. Therefore, the PAPRs in the resulting transmitted signals with MEP are similar to that in $\mathrm{CE}$ precoding, which enables the use of highly non-linear and power efficient PAs for the BS circuitry in practice.

This letter is organized as follows. We describe the system model in Section II. We explain the proposed MEP technique for continuous and discrete PSs in Sections III-A and III-B, respectively, and analyze the complexities of the algorithms in Section III-C. We illustrate the performance of the newly proposed technique via numerical examples in Section IV, and finally conclude this letter in Section V.

\section{System Model and Problem Statement}

A single cell massive MIMO system with a BS equipped with $N$ antennas serving $K$ single antenna users is considered. The channel gain between the $k$-th terminal and the $n$-th BS antenna is denoted by $h_{k, n}$, and the channel vector from all the BS antennas to the $k$-th user is formed as $\mathbf{h}_{k}=\left[h_{k, 1}, h_{k, 2}, \ldots, h_{k, N}\right]^{T}$. The channel matrix between all the BS antennas and all the users is shown by $\mathbf{H} \in \mathbb{C}^{K \times N}$ whose $(k, n)$-th element is $h_{k, n} . P_{B S}$ denotes the total transmitted power, and $\mathbf{p}=\left[p_{1}, p_{2}, \ldots, p_{N}\right]^{T}$ is the power coefficient vector satisfying $\mathbf{p}^{T} \mathbf{p}=N$ to meet the transmit power constraint. The signal emitted from the $n$-th $\mathrm{BS}$ antenna is given by $x_{n}=\sqrt{P_{B S} / N} p_{n} e^{j \theta_{n}}$ where $\theta_{n}$ is the phase of the signal. We refer to $\boldsymbol{\theta}=\left[\theta_{1}, \theta_{2}, \ldots, \theta_{N}\right]^{T}$ as the phase angle vector.

The signal received at the $k$-th user is given by

$$
y_{k}=\sqrt{P_{B S} / N} \sum_{n=1}^{N} h_{k, n} p_{n} e^{j \theta_{n}}+w_{k}, k=1,2, \ldots, K,
$$

where $w_{k} \sim C N\left(0, \sigma^{2}\right)$ is the circularly symmetric complex Gaussian noise term. Let $u_{k} \in U_{k}$ (where $U_{k}$ is the unit energy 
information alphabet) represent the normalized symbol being transmitted to the $k$-th user, and $E_{k}$ be the symbol energy for $k=1,2, \ldots, K$. Then, $\mathbf{u}=\left[\sqrt{E_{1}} u_{1}, \ldots, \sqrt{E_{K}} u_{K}\right]^{T}$ is the scaled information symbol vector, and $\mathcal{U} \triangleq \sqrt{E_{1}} U_{1} \times$ $\sqrt{E_{2}} U_{2} \times \ldots \times \sqrt{E_{K}} U_{K}$ is the (fixed) scaled information symbol library. The received signal at the $k$-th user can be expressed as

$$
y_{k}=\sqrt{P_{B S}} \sqrt{E_{k}} u_{k}+\sqrt{P_{B S}} s_{k},
$$

where

$$
s_{k}=\frac{\sum_{n=1}^{N} h_{k, n} p_{n} e^{j \theta_{n}}}{\sqrt{N}}-\sqrt{E_{k}} u_{k} .
$$

Here, $\sqrt{P_{B S}} s_{k}$ is the multi-user interference (MUI) term.

\section{Proposed Multi-Envelope PRECoding TeChNique}

In the proposed MEP technique, the aim is twofold: 1) to assign the individual antennas to the available envelope levels, 2) to determine the phase angles that minimize the sum of the MUI energies of all the users. To meet the first objective, many methods such as use of the sum of the magnitude squares of the gains from a specific antenna to all the users can be applied for antenna grouping, however, the most effective one is observed to be the use of the result of ZF precoding or regularized ZF precoding. ${ }^{1}$ Briefly, the zero-forcing vector is given by $\mathbf{z}_{f}=\left(\mathbf{H}^{H}\left(\mathbf{H H}^{H}\right)^{-1}\right) \mathbf{u}$ where $\mathbf{H}^{H}$ is the Hermitian of the channel matrix. Then, the transmitted signal vector with APC becomes $\mathbf{x}_{z f}=\alpha \mathbf{z}_{f}$ with $\alpha=\sqrt{P_{B S} /\left(\mathbf{z}_{f}{ }^{H} \mathbf{z}_{f}\right)}$, and the received signal can be expressed as $\mathbf{y}_{z f}=\alpha \mathbf{u}+\mathbf{w}$. In other words, the noise-free received signal $\left(\mathbf{y}_{z f}-\mathbf{w}\right)$ is a scaled version of the information symbol vector, which means that the interference due to the simultaneous transmissions is cancelled out with ZF precoding (as we consider a single cell scenario).

We use the vector consisting the absolute values of $\mathrm{ZF}$ vector's elements to determine $\mathbf{p}$. The group of antennas corresponding to the largest absolute values in this vector are assigned to the largest power coefficient, the next group is assigned to the second largest, and so on. Therefore, we reduce the number of envelopes to a few predefined levels with the help of the ZF solution.

For simplicity, let us assume that $N$ is divisible by the number of envelope levels and groups of equal number of elements are employed. We set $p_{1}^{2}+p_{2}^{2}+\ldots+p_{a}^{2}=a$ to meet the transmit power constraint where $a$ denotes the number of envelope levels. For instance, if 2 envelope levels are utilized, $p_{1}>1$ is considered as the envelope coefficient of the first group of antennas (with the corresponding absolute value of the term in the zero forcing vector being greater than that of the remaining half of the antennas), and $p_{2}=\sqrt{2-p_{1}^{2}}$ is the coefficient for the remaining ones.

\section{A. MEP With Continuous Phase Shifters}

Once the power coefficient vector is determined, for known $\mathbf{p}, \mathbf{u}$ and $\mathbf{H}$ values, we formulate the following nonlinear least

\footnotetext{
${ }^{1}$ Our investigations show that the use of regularized ZF precoding of [7] for antenna grouping achieves almost the same performance with zero-forcing precoding, hence we stick to the latter approach.
}

squares (NLS) optimization problem to compute the phase angle vector $\boldsymbol{\theta}$ that minimizes the sum of the MUI energies:

$$
\begin{gathered}
\boldsymbol{\theta}^{u}=\left(\theta_{1}^{u}, \ldots, \theta_{N}^{u}\right)=\underset{\theta_{i} \in[-\pi, \pi), i=1, \ldots, N}{\operatorname{argmin}} g(\boldsymbol{\theta}, \mathbf{u}), \\
g(\boldsymbol{\theta}, \mathbf{u}) \triangleq \sum_{k=1}^{K}\left|s_{k}\right|^{2}=\sum_{k=1}^{K}\left|\frac{\sum_{n=1}^{N} h_{k, n} p_{n} e^{j \theta_{n}}}{\sqrt{N}}-\sqrt{E_{k}} u_{k}\right|^{2} .
\end{gathered}
$$

The optimization problem in (3) is similar to the one in [3], and in fact, it becomes the same if $\mathbf{p}$ is chosen as an all 1 vector, hence it is expected that the solution at most local minima is small enough if the ratio of $N / K$ is high as in [3]. Therefore, we modify the fast iterative algorithm of [3] to solve this problem. That is, at each iteration, one antenna phase is solved to reduce the value of the objective function, and the algorithm terminates after a predefined number of iterations.

There are $N$ subiterations in each step. Let $r$ denote the iteration count and $q$ be the subiteration count. If $q=N$, the algorithm moves to the $(r+1,1)$-st iteration. Otherwise, it moves to the $(r, q+1)$-th iteration. Let $\boldsymbol{\theta}^{r, q}$ be the set of angles after the $q$-th subiteration of the $r$-th iteration. Then, keeping all the other phase angles the same as those in the previous subiteration, $\boldsymbol{\theta}^{r, q+1}$ is given by

$$
\begin{aligned}
\boldsymbol{\theta}^{(r, \boldsymbol{q}+\mathbf{1})} & =\underset{\boldsymbol{\theta}=\theta_{1}^{(r, q)}, \ldots \theta_{q}^{(r, q)}, \phi, \theta_{q+2}^{(r, q)}, \ldots, \theta_{N}^{(r, q)}, \phi \in[-\pi, \pi)}{\operatorname{argmin}} g(\boldsymbol{\theta}, \mathbf{u}), \\
& =\pi+\arg \left(\sum_{k=1}^{K} \frac{h_{k, q+1}^{*}}{\sqrt{N}}\left[\left(\frac{\sum_{n} h_{k, n} p_{n} e^{j \theta_{n}^{(r, q)}}}{\sqrt{N}}\right)-\sqrt{E_{k}} u_{k}\right]\right),
\end{aligned}
$$

where the inner summation is over all $n=1,2, \ldots, N$ except for $n=q+1$. Also, $\boldsymbol{\theta}^{(\boldsymbol{r}, \boldsymbol{q}+\mathbf{1})}=\boldsymbol{\theta}^{(\boldsymbol{r}, \boldsymbol{q})}$ for all $n=1,2, \ldots, N$ except for $n=q+1$.

Denoting the phase angles after the last iteration as $\hat{\boldsymbol{\theta}}^{u}=$ $\left[\hat{\theta}_{1}^{u}, \ldots, \hat{\theta}_{N}^{u}\right]^{T}$, the MUI at the $k$-th user is given by

$$
\hat{s}_{k}=\frac{\sum_{n=1}^{N} h_{k, n} p_{n} e^{j \hat{\theta}_{n}^{u}}}{\sqrt{N}}-\sqrt{E_{k}} u_{k} .
$$

Finally, the signal to interference and noise ratio (SINR) and the corresponding achievable rate expressions become

$$
\begin{gathered}
\gamma_{k}\left(\mathbf{H}, E, P_{B S} / \sigma^{2}\right)=\frac{E_{k}}{\mathrm{E}_{u_{1}, \ldots, u_{K}}\left[\left|\hat{s}_{k}\right|^{2}\right]+\sigma^{2} / P_{B S}}, \\
R_{k}\left(\mathbf{H}, E, P_{B S} / \sigma^{2}\right)=\log _{2}\left(\gamma_{k}\left(\mathbf{H}, E, P_{B S} / \sigma^{2}\right)\right),
\end{gathered}
$$

respectively, where $\mathrm{E}$ denotes expectation.

A more detailed description of the proposed MEP algorithm for continuous phase shifters can be found in [8].

\section{B. MEP With Discrete Phase Shifters}

The use of digital phase shifters may be advantageous compared to continuous phase shifters in terms of immunity to noise on the voltage control signal, more uniform input-output performance and flat phase values over wider bandwidths [6]. Therefore, we extend the newly proposed MEP technique to this case via a suitable modification of the trellis based constant envelope precoding (TB-CEP) algorithm developed in [4] as well. 
With the use of discrete phase shifters, the optimization problem in (3) is modified to

$$
\begin{aligned}
& \boldsymbol{\theta}^{u}=\underset{\boldsymbol{\theta}}{\operatorname{argmin}} \sum_{k=1}^{K}\left|\sqrt{\frac{P_{B S}}{N}} \mathbf{h}_{k}^{T} \Lambda \boldsymbol{\theta}-\sqrt{E_{k}} u_{k}\right|^{2}, \\
& \text { s.t. } \theta_{n} \in \Theta_{M}, n=1,2, \ldots, N \text {, }
\end{aligned}
$$

where $\mathbf{h}_{k}$ is the channel vector to the $k$-th user, i.e., the $k$-th row of $\mathbf{H}, \Theta_{M}$ is set of phase values supported by the phase shifters (that is, $\theta_{n}=\exp \left(j 2 \pi m_{n} / M\right)$ for $\left.m_{n} \in\{0,1, \ldots, M-1\}\right)$, and $\Lambda$ is an $N \times N$ diagonal matrix with entries corresponding to the elements of the power coefficient vector $\mathbf{p}$.

Such integer programming problems have exponential complexity in the worst case. Therefore, solving the problem in (7) via conventional methods is not suitable for massive MIMO systems where $N$ is high. With this motivation, the problem is transformed into a new one by expanding the square in (7), and ignoring the terms that do not depend on $\boldsymbol{\theta}$, resulting in

$$
\begin{aligned}
\boldsymbol{\theta}^{\boldsymbol{u}}=\underset{\boldsymbol{\theta}}{\operatorname{argmin}} & \sum_{j=1}^{N} \operatorname{Re}\left(\sqrt{\frac{P_{B S}}{N}} \sum_{i=1}^{j-1} p_{j} \theta_{j}^{*} \mathbf{G}_{j, i} p_{i} \theta_{i}-\mathbf{u}^{H} \mathbf{E} \mathbf{H}_{j} p_{j} \theta_{j}\right) \\
\text { s.t. } & \theta_{n} \in \Theta_{M}, n=1,2, \ldots, N,
\end{aligned}
$$

where $\mathbf{G}=\mathbf{H}^{H} \mathbf{H}, \mathbf{H}_{j}$ is the $j$-th column of the channel matrix, and the $K \times K$ diagonal matrix $\mathbf{E}$ includes the user symbol energies. The objective function has $N$ real terms where the $j^{\text {th }}$ term depends only on the first $j$ variables, hence the problem is solvable with a multi-stage approach as in [4]. That is, the last few phase values are considered as states, and the most likely paths for each state for all the stages are kept until the last antenna is taken into account, and all of the phase values are decided jointly similar to an application of Viterbi algorithm.

In the proposed MEP algorithm, with the objective of utilizing the signals of the antennas assigned to higher envelope levels in more of the objective function variables, we also reorder the antennas by considering the zero-forcing criterion (as done in the previous section for antenna grouping) before applying the TB-CEP algorithm. Let $\hat{\mathbf{H}}$ be the reordered channel matrix where the first column is the channel realizations of the antenna with the greatest norm in the zero-forcing vector, and the $N$-th column consists of the channel gains for the antenna corresponding to the least norm. Then, $\mathbf{p}$ has the form of $\left[p_{1}, \ldots, p_{1}, p_{2}, \ldots, p_{a-1}, p_{a}, \ldots, p_{a}\right]^{T}$, where $p_{1}>p_{2}>\ldots>p_{a}$ as we have already reordered the channel matrix. After reordering, the phase angles are determined by using the multi-stage TB-CEP algorithm for known $\mathbf{p}$, $\mathbf{u}$ and $\hat{\mathbf{H}}$.

In the TB-CEP algorithm, the initilization is determined by considering all the possibilities for the phase values, hence there are $M^{L}$ initial states where $L$ denotes the memory in the multi-stage algorithm. The cumulative metrics of all the initial states are calculated as follows

$$
\sum_{j=1}^{L} \operatorname{Re}\left(\sqrt{\frac{P_{B S}}{N}} \sum_{i=1}^{j-1} p_{j} \theta_{j}^{*} \hat{\mathbf{G}}_{j, i} p_{i} \theta_{i}-\mathbf{u}^{H} \mathbf{E} \hat{\mathbf{H}}_{j} p_{j} \theta_{j}\right),
$$

where $\hat{\mathbf{G}}=\hat{\mathbf{H}}^{H} \hat{\mathbf{H}}$. We note that $M$ branches go out of each state and $M$ branches enter at each step. A state is determined by the latest $L-1$ phase values of the originating state and the branch label, which is the phase chosen for the current stage. At the $t$-th stage, the branch metric is given by

$$
\operatorname{Re}\left(\sqrt{\frac{P_{B S}}{N}} \sum_{i=1}^{t+L-1} p_{t+L} \theta_{t+L}^{*} \hat{\mathbf{G}}_{t+L, i} p_{i} \theta_{i}-\mathbf{u}^{H} \mathbf{E} \hat{\mathbf{H}}_{t+L} p_{t+L} \theta_{t+L}\right) .
$$

The cumulative metric is calculated for each branch by adding the branch metric to the originating state metric. The path with the least cumulative metric among the $M$ branches, which enter a state, is chosen as the surviving path, the others are discarded, and the state metric is determined as the cumulative metric of the surviving path. The algorithm continues until the last antenna is accounted for, and the best path, which provides the least objective value is chosen. The transmit phase values are determined from the corresponding branch labels and the initial states. The MUI energy for the $k$-th user is calculated as in (5), and the corresponding SINR and the achievable rate are as given in (6).

A more detailed description of the proposed MEP algorithm for discrete phase shifters can be found in [8].

\section{Complexity Analysis}

The proposed MEP algorithms have two serial stages: antenna grouping or reordering of the channel matrix, and calculation of the transmit phase values to minimize the objective function. In the first stage, calculation of the zero-forcing vector includes a pseudo-inverse of a $K \times N$ matrix of complexity $O\left(K^{2} N\right)$, and a matrix vector product of complexity $O(K N)$. The second part of the algorithm for the case with continuous phases has the same complexity with that of the CE precoding of [3], which is $O(K N)$, hence the overall complexity of the algorithm is polynomial similar to the CE precoding case as the pseudo-inverse of the channel matrix is calculated only once for entire coherence time of the channel. The algorithm for the case with discrete phases has the same complexity with TB-CEP algorithm of [4] (which is $O\left(N^{2} K M^{L+1}\right)$ ) at the second stage, hence it has a polynomial complexity as well.

\section{Numerical Results}

We now study the proposed MEP algorithms and compare them with the CE solutions in terms of the resulting achievable rates and minimum power requirements via numerical examples. The channels are assumed to be independent Rayleigh fading $(C N(0,1))$, and the codebooks are complex Gaussian for all the users with $U_{1}=U_{2}=\cdots=U_{K}=C N(0,1)$. In order to assess the power requirements, an achievable rate level $R\left(\frac{P_{B S}}{\sigma^{2}}\right)=2 \mathrm{bpcu}$ is selected, and for assessment of the achievable rates, the user symbol energies, BS power and receiver noise variances are fixed as $E_{k}=P_{B S}=10$ and $\sigma_{w}^{2}=1$, respectively.

In Fig. 1, the minimum required $\frac{P_{B S}}{\sigma^{2}}$ values for a given achievable rate is illustrated. In order to show the resulting improvement with MEP compared to the CE precoding solution, the cooperative upper bound on the GBC sum-capacity is also plotted [3]. We use $p_{1}=\sqrt{3 / 2}, p_{2}=1, p_{3}=\sqrt{1 / 2}$ as the power coefficients with 3 PAs, and $p_{1}=\sqrt{3 / 2}$ and 


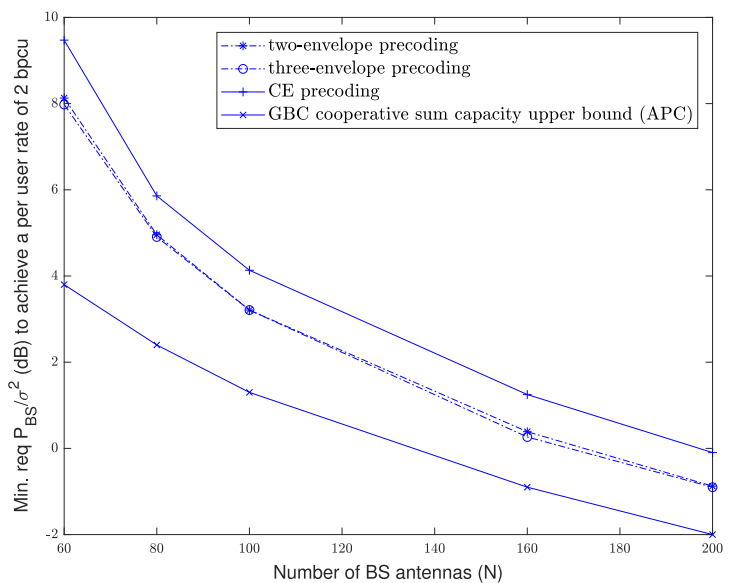

Fig. 1. The required $P_{B S} / \sigma^{2}$ ratio versus the number of antennas. $K=40$, Rayleigh fading.

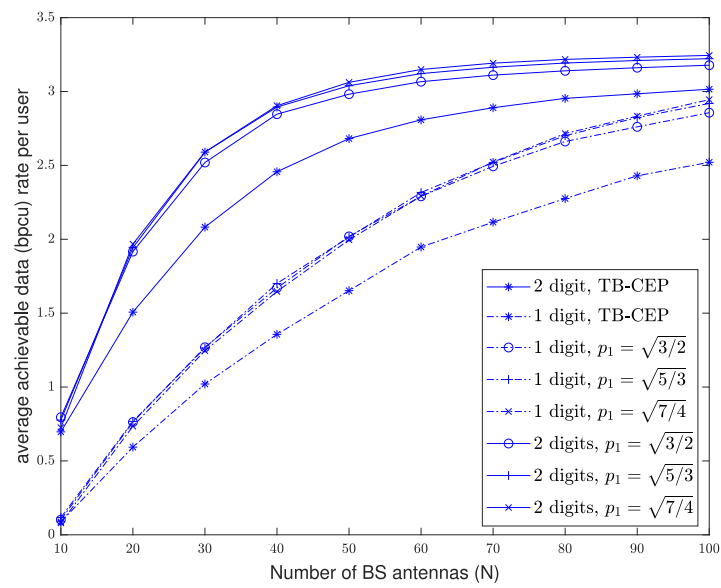

Fig. 2. Average achievable data rate (in bpcu) versus the number of BS antennas over Rayleigh fading. $E_{k}=P_{B S}=10, L=2, K=10, \sigma_{w}^{2}=1$.

$p_{2}=\sqrt{1 / 2}$ with 2 PAs. We observe through Fig. 1 that the extra power required by utilizing the $\mathrm{CE}$ constraint decreases with the use of 2 or 3 PAs and antenna grouping according to the zero-forcing algorithm. For example, for $N=160$, this gain is around $1 \mathrm{~dB}$. Furthermore, it is demonstrated that there is no significant difference between 2 or 3 envelope levels.

In Fig. 2, we simulate the achievable rate performance of the MEP with discrete phase shifters for different number of phases and power coefficients. We observe that the availability of more phase values (through the use of PSs with more digits) improves the achievable data rates, and the newly proposed MEP technique outperforms the TB-CEP algorithm even with 2 PAs. We also observe that the results with $p_{1}=\sqrt{3 / 2}$, $p_{1}=\sqrt{5 / 3}$ and $p_{1}=\sqrt{7 / 4}$ are very similar.

In Fig. 3, we simulate the achievable rate performance of the MEP with discrete phase shifters, TB-CEP and the CE precoding. We observe that the use of discrete phase shifters causes degradation in the performance compared to the case of continuous phase shifters as the number of phases is limited to only a few values. We also observe that, for the present setting, the proposed MEP technique with discrete phase shifters is successful to close the gap between the TB-CEP algorithm and $\mathrm{CE}$ precoding with continuous phase shifters, that is, about

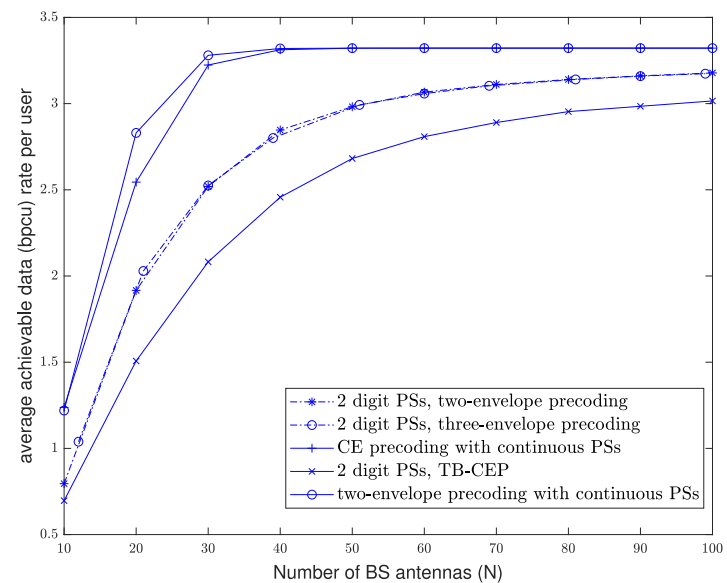

Fig. 3. Average achievable data rate (bpcu) versus the number of antennas over Rayleigh fading. $E_{k}=P_{B S}=10, L=2, K=10, \sigma_{w}^{2}=1$.

half of the gap is removed with the use of only 2-3 PAs when 90-100 base station antennas are utilized at the BS. We also note that, when the user symbol energies are kept constant for all the algorithms, two-envelope precoding with continuous PSs does not provide much improvement as the background noise is dominant as opposed to the remaining MUI energy for this example.

Further results on the performance of MEP are given in [8].

\section{CONCLUSion}

We have proposed a multi-envelope precoding technique for use in massive MIMO systems, which employs only a few envelope levels (with both discrete and continuous phase shifters), one for each BS antenna in an effort to improve the system performance with respect to CE precoding. Our results demonstrate that the MEP technique with as low as 2 envelope levels significantly outperforms the CE precoding and TB-CEP techniques with almost no increase in hardware complexity and only a slight increase in computational complexity.

\section{REFERENCES}

[1] F. Boccardi, R. W. Heath, A. Lozano, T. L. Marzetta, and P. Popovski, "Five disruptive technology directions for 5G," IEEE Commun. Mag., vol. 52, no. 2, pp. 74-80, Feb. 2014.

[2] E. G. Larsson, O. Edfors, F. Tufvesson, and T. L. Marzetta, "Massive MIMO for next generation wireless systems," IEEE Commun. Mag., vol. 52, no. 2, pp. 186-195, Feb. 2014.

[3] S. K. Mohammed and E. G. Larsson, "Per-antenna constant envelope precoding for large multi-user MIMO systems," IEEE Trans. Commun., vol. 61, no. 3, pp. 1059-1071, Mar. 2013.

[4] M. Kazemi, H. Aghaeinia, and T. M. Duman, "Discrete-phase constant envelope precoding for massive MIMO systems," IEEE Trans. Commun., vol. 65, no. 5, pp. 2011-2021, May 2017.

[5] S. K. Mohammed and E. G. Larsson, "Constant-envelope multiuser precoding for frequency-selective massive MIMO systems," IEEE Wireless Commun. Lett., vol. 2, no. 5, pp. 547-550, Oct. 2013.

[6] Microwave101 Phase Shifters. Accessed: Jun. 3, 2017. [Online]. Available: https://www.microwaves101.com/encycl-opedias/phaseshifters

[7] C. B. Peel, B. M. Hochwald, and A. L. Swindlehurst, "A vector-perturbation technique for near-capacity multiantenna multiuser communication-part I: Channel inversion and regularization," IEEE Trans. Commun., vol. 53, no. 1, pp. 195-202, Jan. 2005.

[8] M. Gümüş, "Multi-envelope precoding for massive MIMO systems," M.S. thesis, Elect. Electron. Eng., Bilkent Univ., Ankara, Turkey, Jun. 2017. 